\title{
A system-level simulation framework for LTE Femtocells
}

\author{
[Poster Abstract] \\ F. Capozzi, G. Piro, L. A. Grieco, G. Boggia, P. Camarda \\ DEE - Politecnico di Bari, Bari, Italy \\ \{f.capozzi, g.piro, a.grieco,g.boggia, camarda\}@poliba.it
}

\begin{abstract}
Femtocells represent a new technological advance, conceived to significantly boost cellular network performance. At the same time, several issues that could arise with their deployment, still need to be investigated. Unfortunately, to the best of our knowledge, no accurate simulation tools are freely available for dealing with femtocells. To bridge this gap, we propose herein an open source module implemented within the emerging LTE-Sim framework. It targets heterogeneous scenarios with both macro and femtocells and, thanks to its flexibility, it is well suited to study spectrum allocation techniques, user mobility, femtocell access policies, and several other problems related to femtocell deployment in a LTE network. We claim that this new module can be of high interest for the research community because it offers a very wide range of scenarios and tunable parameters and because, thanks to its high modularity, it can be simply extended for the study of current and future hybrid network architectures.
\end{abstract}

\section{Categories and Subject Descriptors}

I.6.5 [Simulation and Modeling]: Model DevelopmentModeling methodologies; I.6.7 [Simulation and Modeling]: Simulation Support Systems-Environments

\section{General Terms}

Algorithm, Design, Performance, Verification

\section{Keywords}

Femtocell, 3GPP, LTE, E-UTRA, Simulator, Channel, MAC, PHY

\section{INTRODUCTION}

Femtocells represent, nowadays, a low-cost attractive solution for improving both coverage and capacity of broadband cellular networks. Market forecasts are expecting a

Permission to make digital or hard copies of all or part of this work for personal or classroom use is granted without fee provided that copies are not made or distributed for profit or commercial advantage and that copies bear this notice and the full citation on the first page. To copy otherwise, to republish, to post on servers or to redistribute to lists, requires prior specific permission and/or a fee. Simutools 2012, March 19-23, Desenzano del Garda, Italy Copyright $\odot 2012$ ICST 978-1-936968-47-3 DOI 10.4108/icst.simutools.2012.247767 massive increase in data traffic generated by mobile users in upcoming years so that emerging broadband technologies (i.e., WiMAX, LTE, and LTE-A) require significant architectural improvements to become able to satisfy this ever growing user demands. In this perspective, the support of high data-rate indoor traffic becomes fundamental, since it is estimated that most of the voice calls and data sessions will take place in indoor environment during the next years [8]. The deployment of cellular network composed by very small cells (i.e., femtocells) represents today the smartest solution for boosting cellular network services, especially in indoor scenarios [3]. A femtocell in LTE environment is obtained using a Home evolved Node B (HeNB), that is a low-power and small-range cellular base station, typically designed for home or small business environment. It has plug-and-play capabilities to be straightly set up by end-users; moreover, it is connected to the core network through the DSL line available at consumers' houses or offices.

Several benefits are expected from deployment of femtocells, starting from an increased network capacity [3]. The market of femtocells is expected to be very attractive for both mobile operators and consumers. From one side, they can guarantee a better coverage to those areas which are currently provided with an unsatisfactory service if served by macrocells. On the other hand, users could take advantage from the reduction of costs related to their generated traffic. According to [7], in fact, the cost per MByte of the traffic handled inside a femtocell is expected to be less than the one managed by a macrocell. Nevertheless, several issues have still to be investigated, especially in the area of the Radio Resource Management (RRM). Among others, interference management, as matter of fact, becomes a major issue in conditions of uncoordinated deployment of HeNBs. In fact, when the operator cannot perform a centralized frequency planning, both co-layer (femto-to-femto) and crosslayer (femto-to-macro) interference take place. This problem appears even more complex when restricted access policies are used at HeNBs.

Therefore, the availability of accurate simulation frameworks, able to correctly modeling and test femtocell scenarios, appears of fundamental importance. Unfortunately, while several open-source tools are today available for simulating some parts of the LTE system (i.e., [4] and [2]), none of them provides MAC and PHY models for femtocell architectures.

To bridge this gap, we propose herein a new module for simulating LTE femtocells within the opensource LTE-Sim framework [6]. The developed tool provides a wide range 
of features and high scalability. It encompasses scenarios with both macrocells and femtocells in multi-cell and multiuser environments, taking into account a complete LTE protocol stack, as well as advanced RRM techniques, such as frequency reuse schemes, packet scheduling, and QoS management. User mobility is also considered and several configurations of mobility patterns and handover schemes are covered. We believe that it represents a good research tool for tackling several open issues.

\section{FRAMEWORK DESCRIPTION}

LTE-Sim ${ }^{1}$ has been written in $\mathrm{C}++$, using the objectoriented paradigm, as an event-driven simulator [6]. Its main classes handle: (i) network devices, (ii) protocol stack entities, (iii) PHY layer, (iv) channel behavior, and (v) network topology. In order to implement all aspects related to femtocells, LTE-Sim has been upgraded by means of new classes and functionalities listed below:

- Network devices. A new class has been defined to model the HeNB, which is identified by an unique ID and its position defined into a Cartesian System. A HeNB can be configured for working in both open access and closed access modes. Moreover, attached to it, a proper physical interface accounts for the different power management of suchdevices with respect to conventional eNBs. User equipments, on the other hand, have been enhanced for recognizing whether they are inside or outside a building at a certain time instant and for catching propagation losses due to walls between the transmitter and the receiver.

- New handover management. A power-based handover manager has been conceived for better handling handover procedures in heterogeneous network. Such a mechanism is aware of the selected access policy at the HeNB, in order to avoid re-selections of unauthorized cell.

- New topology objects. Three new network topology objects have been introduced: Femtocell, Building, and Street. The class Femtocell is in charge of storing geometrical parameters of the cell. A Building is composed by a number of apartments, each one delimiting the area of a given femtocell. As suggested in [1], two different types of building have been developed: (a) Dual Stripe blocks and (b) $5 \times 5$ apartment grid. Furthermore, in order to be able to design a wide variety of urban scenarios, we introduced the concept of Street, consisting of a double row of buildings.

- New channel models. To cope with the characteristics of indoor environments, we implemented two new indoor propagation loss models. The former has been proposed in [1] for femtocell dense urban scenarios. The latter, instead, developed within the WINNER II project, is more suitable for indoor residential environments [5].

\section{NUMERICAL RESULTS}

${ }^{1}$ The code is freely available under the GPLv3 license. URL: http://telematics.poliba.it/LTE-Sim

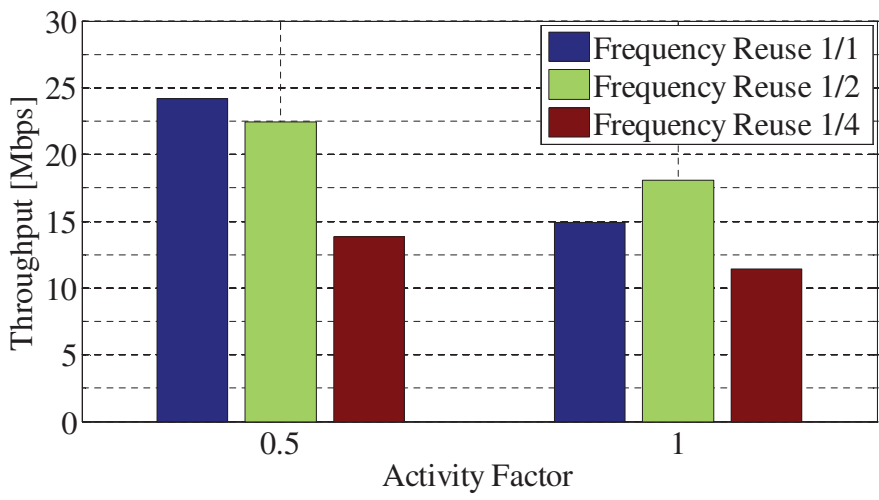

Figure 1: Aggregate throughput of the building.

In this section, some reference results are presented to highlight the potentials of the simulator we conceived. We evaluated: (i) the impact of co-layer interference in indoor environment, (ii) the performance gains coming from femtocell deployment in an urban scenario, and (iii) a scalability study on computational requirements (i.e., simulation time and memory usage). Main simulation parameters are summarized in Tab. 1.

Fig. 1 shows aggregate cell throughput in a single $5 \times 5$ grid building, considering different activity factors (i.e., the probability that a single femtocell is active) and frequency reuse schemes. In details, under Reuse- 1 , an overall bandwidth of $20 \mathrm{MHz}$ is shared among all the femtocells in the building; under Reuse- $1 / 2$ and Reuse- $1 / 4$, instead, the entire $20 \mathrm{MHz}$ bandwidth is partitioned in two and four portions of spectrum, respectively. Results demonstrate that when the interference level is low (i.e., activity factor is equal to $50 \%$ ), Reuse- 1 scheme performs better, as the SINR is not heavily impacted and each cell exploits the $20 \mathrm{MHz}$ band for data transmission. On the other hand, as the impact of interference increases, Reuse-1 scheme suffers for strong SINR degradation and consequent performance drop. Reuse- $1 / 2$ scheme, in this context, appears to be the best trade-off between higher bandwidth availability (i.e., Reuse-1) and higher experienced SINR (i.e., Reuse-1/4).

To evaluate network capacity enhancements due to femtocells deployment, we designed a dense urban environment consisting of a macrocell and of 56 buildings located as in a typical urban cross. Two different cases are object of the study: (i) a scenario where buildings without femtocells are distributed into a macro, and (ii) the same topology where one femtocells per apartment is assumed to be active and working on the same operative bandwidth of the macrocell. Fig. 2 shows the the aggregate network throughput. It demonstrates that the introduction of femtcells into a urban scenario improves network performance. Without femtocells, in fact, indoor users will be anyway served by the macrocell suffering for low channel quality due to the strong influence of walls attenuation. Introducing femtocells, indoor users will experience a significant increase of the throughput.

Finally, we studied the scalability of the proposed module on a Linux machine with a $2.6 \mathrm{GHz} \mathrm{CPU}$ and 4 GBytes of RAM. In particular, we evaluated the impact that the number of buildings, as well as the number of users created inside each building, have on both simulation time and mem- 


\begin{tabular}{l|c|c|c}
\multicolumn{2}{c}{ Table 1: Simulation Parameters. } & urban scenario & scalability test \\
\hline Parameter & indoor environment & $100 \mathrm{~m}^{2}$ & $100 \mathrm{~m}^{2}$ \\
\hline Apartment size & $100 \mathrm{~m}^{2}$ & 25 & 25 \\
Number of apartment in a building & 25 & 56 & from 1 to 6 \\
Number of buildings & 1 & $500 \mathrm{~m}$ & $500 \mathrm{~m}$ \\
Radius of the Macro cell & N.A. & from 40 to 120 & from 0 to 300 \\
Number of users & 2 for each HeNB & $20 \mathrm{MHz}$ & $20 \mathrm{MHz}$ \\
Total Bandwidth & $20 \mathrm{MHz}$ & Reuse-1 & Reuse-1 \\
Frequency Reuse Scheme & Reuse-1,Reuse-1/2,Reuse-1/4 & 1 & 1 \\
Activity Factor & 0.5 and 1 & Proportional Fair & Proportional Fair \\
Scheduler & Proportional Fair & Infinite buffer & Infinite buffer \\
Traffic & Infinite buffer & \multicolumn{2}{c}{}
\end{tabular}

Table 2: Scalability Test.

\begin{tabular}{lc|c|c} 
& & 1 building & 6 buildings \\
\hline \hline Simulation Time [minutes] & 10 UEs for building & 1.7 & 3.19 \\
& 50 UEs for building & 7.6 & 30 \\
\hline Memory Usage [MBytes] & 10 UEs for building & 263 & 460 \\
& 50 UEs for building & 895 & 2050 \\
\hline \hline
\end{tabular}

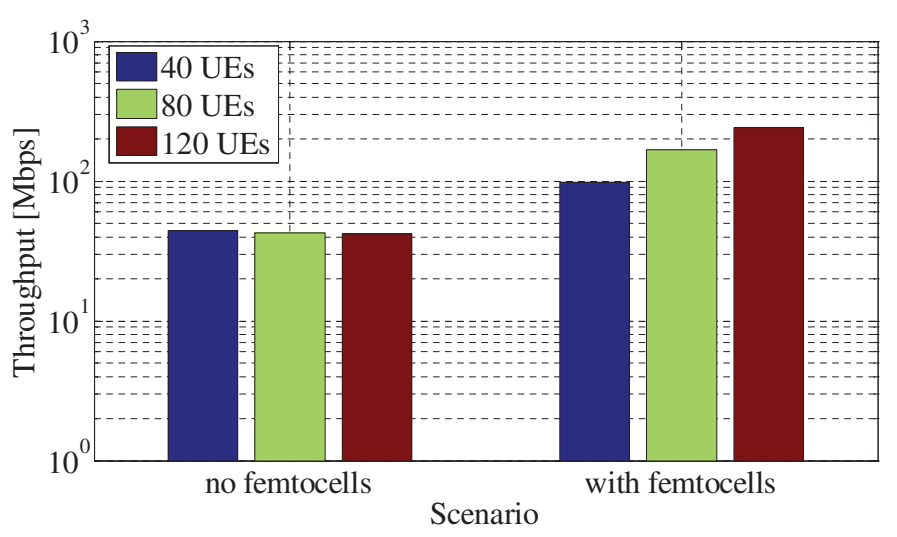

Figure 2: Aggregated throughput in an urban scenario.

ory usage. Obtained measurements (some significant results are reported in Tab. 2) have highlighted that the simulation time, as well as the memory usage, linearly increases with both the number of buildings and the number of users. Moreover, also in the most complex scenario (i.e., the one composed by 6 buildings with 50 users each) computational requirements are quite limited, which is a great achievement in terms of scalability.

\section{CONCLUSIONS AND FUTURE WORKS}

We presented a novel module for simulating LTE femtocell within the LTE-Sim open-source framework. In order to show its potentials, we used the conceived tool for evaluating the impact of co-layer interference in an indoor environment and the performance gain coming from femtocell deployment in an urban scenario. Finally, a scalability study on computational requirements has been also proposed. Preliminary results demonstrate that the implemented module can greatly support people working on LTE femtocells. More- over, thanks to its modularity, it can be upgraded in a very easy way, thus allowing both researchers and industries to design and test innovative algorithms, protocols, and network architectures for next generation mobile systems.

\section{ACKNOWLEDGMENTS}

This work was partially funded by Projects PS025 and A.Q.P. "Modelli Innovativi per Sistemi Meccatronici," Del. CIPE 20/04, DM01, Apulia Region (Italy).

\section{REFERENCES}

[1] 3GPP. R4-092042, Simulation assumptions and parameters for FDD HeNB RF requirements, $3 G P P$ TSG RAN WG4 Meeting 51, May 2009.

[2] N. Baldo, M. Miozzo, M. Requena-Esteso, and J. Nin-Guerrero. An open source product-oriented lte network simulator based on ns-3. In ACM Proc. of Int. Conf. on Modeling, analysis and simulation of wireless and mobile systems, MSWiM, 2011.

[3] V. Chandrasekhar, J. Andrews, and A. Gatherer. Femtocell networks: a survey. IEEE Comm. Mag., 46(9):59-67, Sep. 2008.

[4] J. C. Ikuno, M. Wrulich, and M. Rupp. System level simulation of LTE networks. In Proc. of IEEE Veh. Technol. Conf., VTC Spring, Taipei, Taiwan, May. 2010.

[5] IST-Winner II. WINNER II Channel Models Deliverable D1.1.2 V1.2, Sept. 2007.

[6] G. Piro, L. Grieco, G. Boggia, F. Capozzi, and P. Camarda. Simulating LTE Cellular Systems: An Open-Source Framework. IEEE Trans. Veh. Technol., 60(2):498 -513, Feb. 2011.

[7] Signals Research, LLC. Femtocell Business Case. Femto Forum White Paper, February 2010.

[8] The Femto Forum. The Best That LTE Can Be - Why LTE Needs Femtocells. Femto Forum White Paper, May 2010. 